\title{
Statistical Analysis of Coins Lost in Circulation and Coins Carried
}

\author{
Gregory G. Wood \\ California State University Channel Islands, Camarillo, CA 93012 \\ Tel: 1-805-437-3293 E-mail: gregory.wood@csuci.edu
}

Received: August 13, $2010 \quad$ Accepted: September 5, $2010 \quad$ doi:10.5539/ijef.v3n2p45

\begin{abstract}
Vastly more coins are produced each year than are returned to banks. In this work, the rate at which coins fail to cycle through the economy (the rate of what will be called "lost" coins) is described by a power law with a universal negative exponent meaning coins of high value are less likely to be lost. This power law is then used in a simple statistical model of coin use which is employed to fit prior experimental results: the distribution of number of coins carried.
\end{abstract}

Keywords: Statistical analysis, Coins Carried, Coins Lost

\section{Introduction}

Coins play an essential role in commerce. The production of coins of small value is expensive and shortages of coins are costly to banks and businesses (Gadsby 1996), and contribute to inflation (Chen 1976). Since, generally, the purchasing power of a currency decreases over time, at some point coins of small value should be discontinued and/or new coins of higher value should be issued.

For example, Australia ceased minting two and one cent coins in 1989 and 1990 respectively and began to remove them from circulation in 1992. How coins are carried, used and lost can be a significant input to such decisions. Mints produce vastly more coins than are cycled through the economy and the loss rate is much higher for coins of smaller value (Gadsby 1996; Goldin 1985). Many studies have made attempts to derive superior coin systems in which fewer coins per transaction are required (Bounie 2007; Kelber 2003; Tschoegl 1997). Others study numbers of coins in circulation of various types (Cainiello 1982). These studies do not consider the use and loss of coins by consumers which are discussed in this paper.

\subsection{Prior Estimate of Coin Loss}

A study of the Israeli pound currency, which existed from 1960 through 1979 (Goldin 1985) surveyed a sample of the coins returned to banks after the end of production and found that coins are lost over time and coins of small value are much more likely to be lost then coins of high value.(Note 1) The total loss rate (Table four in Goldin's original paper (Goldin 1985)) is one minus the number of coins estimated to survive at the end of 1979 divided by the total number of coins produced. Although the number of coins produced is well known, the number surviving has to be estimated. This estimate is based on a sample of about 6000 coins which were returned to the mint after the currency was removed from circulation. To distinguish this loss rate from the other (see below) this is called loss rate A. Loss rate A versus coin value are presented in Fig. 1 below and roughlyfollow a power law of the form:

$$
r=a v^{b}
$$

where $r$ is the loss rate, $v$ is the coin value (which, in order to compare with other currencies need be adjusted for inflation and conversion), $\mathrm{a}$ is the prefactor and $\mathrm{b}$ is the exponent. In general, the exponent is found to be negative meaning the coins of high value are less likely to be lost.

\subsection{A Simple Alternative Estimate of Coin Loss}

A similar loss rate, which is hereafter referred to as loss rate B for clarity, can be attained via a simpler method and yields remarkably similar results. To understand this method it is necessary to briefly describe the ideal life cycle of a coin. At the beginning, the coin is minted and delivered to a bank which distributes them (mostly) to businesses, which use them to make change for consumers. Then the consumer would either use the coin at a business or perhaps bundle the coin with others and return them to a bank. The businesses themselves also return coins to the banks after use. The banks then return the coins to agencies of central banks. At this stage, each coin is examined for fitness and damaged coins are discarded while the rest are re-issued. After many cycles through the economy, the well used coin would be melted down and recycled. In such an ideal system, the number of coins produced by the mint would roughly equal the number destroyed - any excess production would reflect growth in the economy. 
Reality is quite different: most coins are lost far before they are damaged. A circulation rate can be calculated as the number of coins returned to the central bank divided by the number put into circulation (both good coins returned and new coins issued comprise this number). The circulation rate for one cent coins in the US was 0.34 in 1995 (Gadsby 1996). Euro cents fared somewhat better: in 2008, their circulation rate was 0.55 (European Central Bank 2010).

One minus the circulation rate is a measure of loss rate of coins, here called loss rate $\mathrm{B}$, which parallels the loss rate of coins found previously (loss rate A) and is also plotted in Fig. 1. Although generally loss rate A has a higher $\mathrm{y}$-intercept (higher rates of loss) then loss rate B, this is because loss rate A is calculated over the lifetime of the currency instead of a single year.

Coins returned to central banks are survivors in loss rate B, but only coins which endure to the end of the lifespan of the currency (perhaps many years) are survivors in loss rate A. Thus the same coin could be counted as "not lost" many times in method B, but only once in method A.

In order to compare various currencies over time, it is necessary to employ conversions from one currency to another, and to account for inflation over time. The unit of currency used in Fig. 1 is the 2008 one cent (US) coin. All such factors are given in Table 1. The exponent in the power law fit (slope in Fig. 1) is independent of these factors, which can be absorbed into the prefactor (y-intercept in Fig. 1).

\section{Application to Coins Carried}

\subsection{Survey of Coins Carried and a Model of Coin Use}

The Euro Spatial Diffusion Observatory surveyed the process of diffusion of euro coins across France for three years after the introduction of the Euro in 2002. Since coins are stamped with nation of origin, the main thrust of the work was to study the diffusion of currency across nations (Grasland 2002) however it also produced a distribution of number coins carried: a sharply peaked distribution with a long exponential tail showing a peak at 10 coins and an average number of coins carried of about 14 (see Fig. 2 below).

Fits to the data were achieved (Nuño 2005) employing a model of coin use as follows: each pocket starts empty and undergoes about 5000 transactions to reach equilibrium. Transaction amounts are random and range from one cent up to one minus the value of the smallest banknote. For the Euro, this range is from one to 499 cents, since the smallest banknote is worth five Euros and there are one hundred cents per Euro. If insufficient change is in the pocket to pay the bill, the lowest banknote is used instead. If exact change is available, it is given. Otherwise a combination of coins are used the value of which exceeds the transaction value by the minimum amount.

There is often more than one way to pay a certain amount and so with probability $p$ the bill is paid with the minimum number of coins, and with probability q the bill is paid with the maximum. With this algorithm, best fits are attained with $\mathrm{q} \approx 0.63$ (Nuño 2005).

\subsection{A Model of Coin Use Accounting for Coin Losses}

In this work, an alternative model is proposed which integrates the power law form of loss rates described above. Just as in the model described above, transaction values are random and have the same range. There are two cases to consider: either the pocket contains sufficient change to pay the bill or not. In the second case, the smallest banknote with value above the value of the transaction is used, and change is returned. Whenever change is about to be added to the pocket, the power law probability is consulted to check if the coin is lost.

In the first case, sufficient change exists to pay the bill. If exact change is available, it is paid with the minimum number of coins possible. Otherwise, the bill is overpaid by the lowest amount possible using at most two coins. Again, any change returned is checked via the power law loss rate to ensure it gets to the pocket.

After each transaction one of two outcomes can occur: either the pocket is measured or another transaction occurs. Let the probability of going on to another transaction be denoted by $q$ and the probability of measurement be $p$ where $\mathrm{p}+\mathrm{q}=1$. This gives rise to the geometric distribution of number of transactions before measurement produced in Fig. 3.

In total, this model has three adjustable parameters. Two parameters account for the odds of losing a coin as a function of coin value in power law form (prefactor a and exponent $b$ ) and these are confined to the fit of the Fig. 1 loss rates within a one sigma variance from best fit parameters. The final parameter is the probability q of continuing to another transaction before being measured.

One of the papers dealing with the spacial diffusion of coins (Grasland 2002) note that about 13\% of people surveyed carried no coins. Both models (the original from the paper, Nuño (2005) and the new model with coin loss presented here) only account for the remaining respondents who were carrying coins. 
Assumedly some people had been carrying change and spent it all (this would be rather rare) and others simply choose not to carry change. Thus all pockets without coins automatically undergo another transaction. Poisson noise was assumed to be the only source of uncertainty in the survey data, meaning the one sigma uncertainty is the square root of the number of coins. Best fits (plotted as squares in Fig. 2) are achieved with a q value of 0.85 , a power law exponent of $b=-0.37$ and prefactor of $a=70.2$.

For the purposes of analyzing the goodness of fit, and the probability of each transaction occurring, $10^{5}$ pockets are simulated and twenty times as many are employed to produce the data for Figs. 2 and 4.

\section{Discussion}

\subsection{Loss Rates}

In Fig. 1, the high coin value data (greater than 10 cents US, 2008) are generally well fit with a power law. It is difficult to draw any distinction between data due to relatively large error bars. Low value coin data reveal a trend in which the Euro is the least frequently lost coin, followed by the US coins, with the Israeli coins most frequently lost. Since the Israeli coin loss rate is calculated in a different manner (loss rate A), it is not possible to compare with the others directly. However there seems to be a firm result that the US coins are lost at a higher rate than Euro coins. Since US households produce about $45 \%$ more solid waste by mass than those in the EU (deTilly 2004), such a result is not unexpected.(Note 2) Only the exponent seems universal which means the prefactor could be used as a measure of the relative value of the currencies (or perhaps more accurately stated the lack of value). If two nominally different coins in two different nations are lost at the same rate, they are equal in the attention people pay them, if not in value. This suggests a conversion between currencies which may differ from the face value or other possible translations between currencies (such as purchasing power parity).

\subsubsection{Consistency of Loss Rates}

To examine the consistency of the loss rate, data for the Euro is used for the years 2008, 2009 and 2010 (January to July only). All power law fit parameters for all years are consistent with one another to within one standard deviation, as shown in Table 2.

\subsubsection{Uncertainties of Loss Rates}

The uncertainty of the loss rates are derived from slightly different methods. For loss rate A, it can be estimated by the Poisson statistics of the roughly 6000 examined in the study by Goldin (1985). This yields the 2.0 to $5.6 \%$ uncertainties shown in Fig. 1 on the triangles. For loss rate B data, the Poission errors are tiny, since on the order of 200 million coins of each denomination are returned to central banks per month. However the month-to-month fluctuation is large and results in about 3\% uncertainty for an average over a twelve month which are the error bars on the squares and circles in Fig. 1. Further, examining year to year fluctuations yields about the same 3\% result. The US coins are somewhat arbitrarily assigned the same 3\% uncertainties in Fig. 1 since they were produced in similar quantities to the Euro coins and due to lack of any other data.

\subsection{Distribution of Number of Coins}

For the purposes of fitting the distribution of coins carried, the power law from the loss rates is employed. Since these fits are not perfect, the prefactor and exponent are allowed to vary within the one sigma uncertainties resulting from the power law fit to all coin data which are for the prefactor: $a=71.07 \pm 4.77$ and for the exponent: $b=-0.411$ \pm 0.045 . The best fits to the distribution of coins carried are achieved with a prefactor of $a=70.2$ and an exponent of $b=-0.37$. If these parameters are less restricted, the exponent will fall to a small value while the goodness of fit (chi-squared) improves by only about $10 \%$. If coin loss is removed from the model the fits are far worse with a chi-squared about three times larger.

The denominations of coins carried are displayed in Fig. 4. Many more low value coins are carried since the large value coins are virtually always used to pay down large bills. In fact, no more than two of the two-euro coins will ever be present in this model since any bill which would return a two-euro coin as change (and thus has a value under 300 cents) could be overpaid using the two-euro or smaller coins, which reduces the possible change returned to one euro or less. For the same reason, no more than four of the one euro coins will be present.

In Fig. 2, the number of coins carried in the 30-40 coins-per-pocket region is not well fit. This region is also underestimated by the model of the original paper (Goldin 1985). Only the region from three to 30 coins-per-pocket is included in the fit. This indicates the model does not account well for the relatively small number of people carrying large (greater then about 30) coins. It is easy to extend this model to better fit this greater than 30 coin carrying population at the expense of additional parameters. 


\section{Conclusion}

The rate at which coins are lost is fit by a power law with a negative exponent as a function of the value of the coin. Differences are detected between different regions, with the US having higher loss rates then the EU, however the exponent of the power law (slope of Fig. 1) shows remarkable consistency across three disparate currencies and two different methods of calculating loss rates. The best fit parameters are stable from year to year, as illustrated in Table 2. The value of the exponent, about -0.4 , means that doubling the value of the coin reduces the loss rate by a factor of 0.75 . This loss rate, together with a model of coin use, can account for the distribution of coins carried from a prior survey (Goldin 1985). The model predicts the quantities of each of the eight varieties of Euro coins carried (see Fig. 4).

\section{References}

Bounie, D. \& Houy, N. (2007). What Europe Needs is an 80 Cents Coin. École Nationale Supérieure des Télécommunications. Working Paper ESS-07-21.

Caianiello, E.R., Scarpetta, G. \& Simoncelli, G. (1982). A Systemic Study of Monetary Systems. International Journal of General Systems, 8(1): 81-92.

Chen, C. (1976). Currency Denominations and the Price Level. J. of Political Economy, 84(1): 179-184.

deTilly, S. (2004). Waste Generation and Related Policies: Broad Trends Over the Last Ten Years. Chapter 2 from: Addressing the Economics of Waste, OECD.

European Central Bank Statistics.

(2010).

https://stats.ecb.europa.eu/stats/download/bkn_coins_quant/bkn_coins_quant/bkn_coins_quant.zip.

Gadsby, J.W. (1996). Future of the Penny. United States General Accounting Office, http://www.gao.gov/archive/1996/gg96153t.pdf .

Goldin, E. (1985). Statistical Analysis of Coins Lost in Circulation. J. Bus Econ Stats. 3(1): 36-42.

Grasland, C., Guèrin-Pace, F. \& Tostain, A. (2002). The circulation of euros as a reflection on people mobility. Population and Societies. 384(1): 1-4.

Kleber, M., Vakil, R. \& Shallit, J. (2003). What this country needs is an 18c piece. The Mathematical Intelligencer. 25(2): 20-23.

J.C. Nuño, C. Grasland, F. Blasco, F. Guerin-Pace, J. Olarrea, \& B. Luque. (2005). How many coins are you carrying in your pocket? Physica A. 354(1): 432-436.

Tschoegl, A.E. (1997). The optimal denomination of currency, Journal of Money, Credit and Banking. 29(4): 546-554.

United States Bureau of Labor Statistics. (2010). Consumer Price Index Calculator, http://www.bls.gov/data/inflation_calculator.htm.

\section{Notes}

Note 1 . The study also found a correlation to coin diameter: larger coins were less likely to be lost. This is not considered in the present work.

Note 2. This is not to say the lost coins can all be found in landfills. There is evidence that few coins end up in landfills - see the environmental impacts subsection of the GAO report, Gadsby (1996). Although both the GAO report (Gadsby 1996) and Goldin (1985) reject some possibilities, where lost coins go is uncertain. 
Table 1. Currency conversion factors read as from currency is worth (value) of to currency to adjust for both differences in currency and inflation over time. Note that these conversion factors affect the prefactor of the power law fit in Fig. 1, but not the exponent (slope), which seems universal.

\begin{tabular}{|cccccc|}
\hline From Currency & From Year & Value & To Currency & To Year & Reference \\
\hline US Dollar & 1970 & 5.6 & US Dollar & 2008 & US BLS (2010) \\
\hline US Dollar & 1991 & 1.7 & US Dollar & 2008 & US BLS (2010) \\
\hline US Dollar & 1995 & 1.5 & US Dollar & 2008 & US BLS (2010) \\
\hline US Dollar & 2003 & 1.2 & US Dollar & 2008 & US BLS (2010) \\
\hline Israeli Pound & 1970 & 0.33 & US Dollar & 1970 & Goldin (1985) \\
\hline Euro & 2003 & 1.1 & US Dollar & 2003 & ECB (2010) \\
\hline Euro & 2008 & 1.5 & US Dollar & 2008 & ECB (2010) \\
\hline
\end{tabular}

Table 2. Fit parameters for the Euro currency to the power law loss rate of equation one for the years 2008-2010. The parameters are consistent from year to year meaning they generally fall within or close to the given one sigma standard deviations of one another.

\begin{tabular}{|ccc|}
\hline Year & Prefactor & Exponent \\
\hline 2008 & $54.7 \pm 4.5$ & $-0.32 \pm 0.04$ \\
\hline 2009 & $50.9 \pm 4.3$ & $-0.37 \pm 0.05$ \\
\hline 2010 & $55.6 \pm 4.7$ & $-0.36 \pm 0.05$ \\
\hline
\end{tabular}

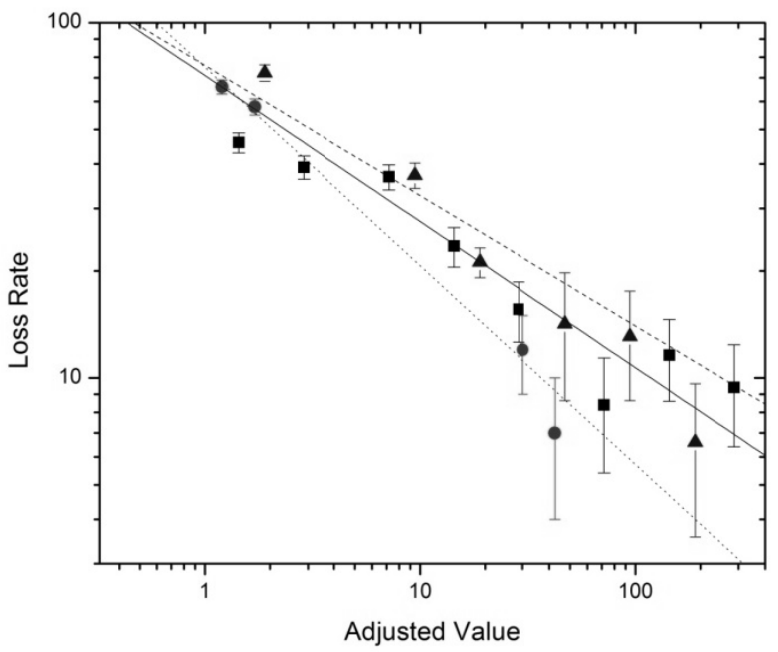

Figure 1. Loss rate as a percentage versus coin value in 2008 US cents (converted via factors in Table 1) for three currencies: US dollar (1993 and 1996) (circles), Euro (2008) (squares) and Israeli Pound nominally 1970) (triangles). Coin values are adjusted for inflation and converted via exchange rates to equivalent 2008 US cents via conversion factors found in Table 1. Loss rates are computed via slightly different methods described in the text as loss rates A (used for only the Israeli data) and B (US and Euro data). Solid line indicates best fit power law to all points; dashed line indicates power law used to fit prior survey results on number of coins carried per person as described below. The best fit power law exponents for each individual currency all fall within $0.40 \pm 0.10$, but the prefactors are significantly different: $101 \pm 7$ for the Israeli Pound, $74 \pm 2$ for the US cents and $55 \pm 5$ for the Euro. 


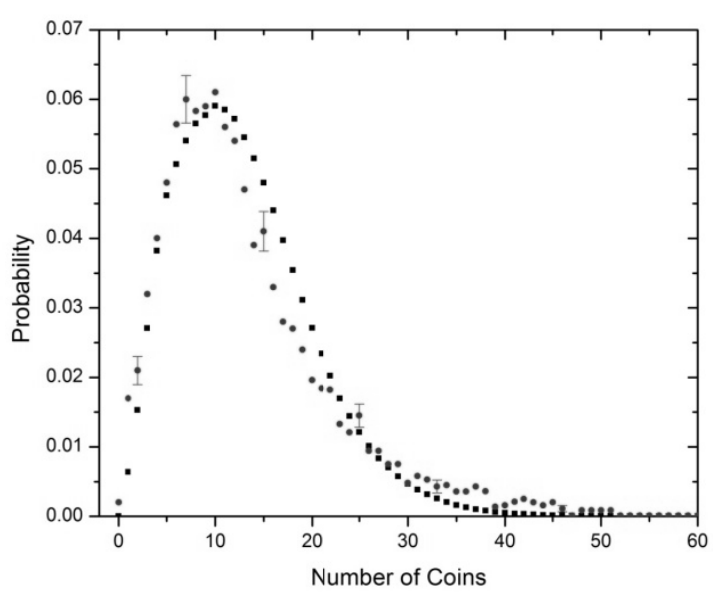

Figure 2. Probability of the pocket containing a certain number of coins versus number of coins carried as found via survey (circles) and calculation (squares) of the model presented in this work which explicitly accounts for the loss of coins via a power law. Similar calculation results have been published Nuño (2005) yet the purpose here is not to compare results but to note that similar results can be achieved while accounting for coin losses. Error bars on select survey results are Poission uncertainties.

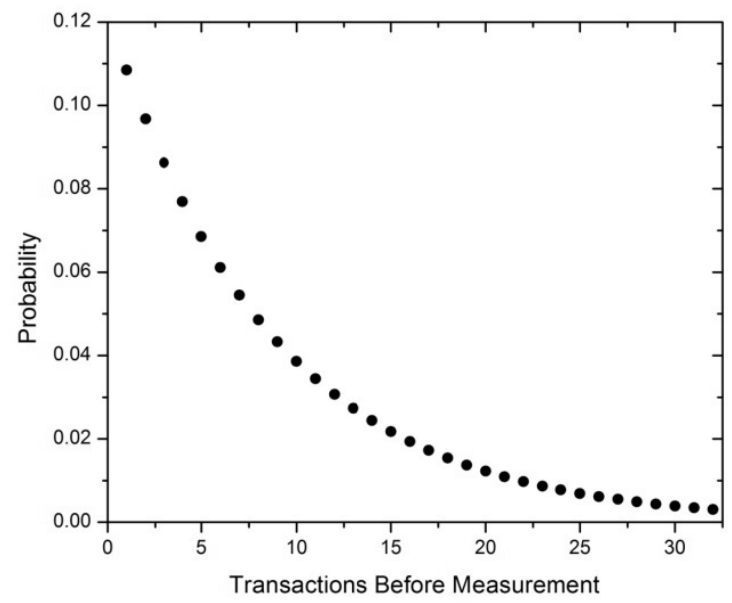

Figure 3. Probability of a particular number of transactions occurring before the measurement versus number of transactions before measurement: a geometric distribution with $\mathrm{p}=0.1085$ the best fit parameter from Fig. 2 above. The average number of transactions before measurement is about 9.2 since at least one transaction must occur before measurement. About a third of survey respondents reported no coins carried and following the original work, no attempt is made in this work to model this behavior. 


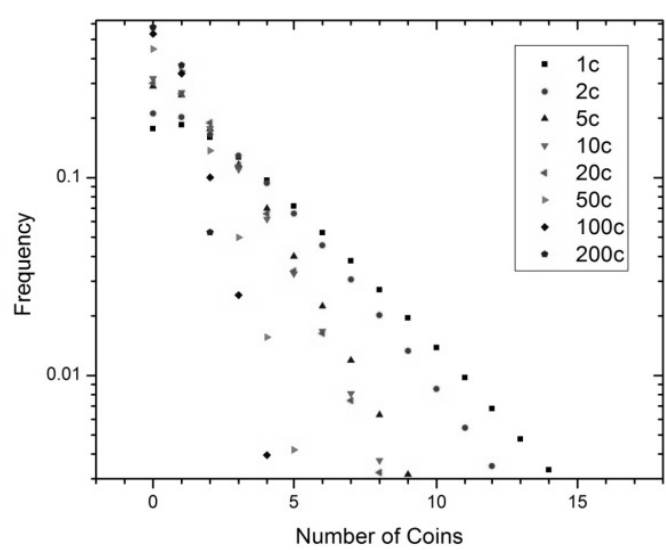

Figure 4. Probability versus number of coins of the eight denominations of Euro coins. Lower value coins occur more often in large numbers. The expected value of coins of each denomination are: one cent (squares) 2.83 , two cent (circle) 2.56, five cent (upright triangle) 1.88, ten cent (inverted triangle) 1.61, twenty cent (left pointing triangle) 1.66 , fifty cent (right pointing triangle) 0.86 , one euro (diamond) 0.63 and two euro (pentagon) 0.48 . 